\title{
Exploring Foraging Decisions in a Social Primate Using Discrete-Choice Models
}

\author{
Harry H. Marshall, ${ }^{1,2,}$ Alecia J. Carter, ${ }^{1,3}$ Tim Coulson, $^{2}$ J. Marcus Rowcliffe, $^{1}$ and Guy Cowlishaw ${ }^{1}$ \\ 1. Institute of Zoology, Zoological Society of London, Regent's Park, London, NW1 4RY, United Kingdom; 2. Division of Ecology and \\ Evolution, Department of Life Sciences, Imperial College London, Silwood Park, Ascot, Berkshire, SL5 7PY, United Kingdom; 3. Fenner \\ School of Environment and Society, Australian National University, Acton, Canberra, Australian Capital Territory, Australia 0200
}

Submitted February 10, 2012; Accepted May 23, 2012; Electronically published August 20, 2012

Online enhancement: appendixes. Dryad data: http://dx.doi.org/10.5061/dryad.8m405.

ABSTRACT: There is a growing appreciation of the multiple social and nonsocial factors influencing the foraging behavior of social animals but little understanding of how these factors depend on habitat characteristics or individual traits. This partly reflects the difficulties inherent in using conventional statistical techniques to analyze multifactor, multicontext foraging decisions. Discrete-choice models provide a way to do so, and we demonstrate this by using them to investigate patch preference in a wild population of social foragers (chacma baboons Papio ursinus). Data were collected from 29 adults across two social groups, encompassing 683 foraging decisions over a 6-month period and the results interpreted using an information-theoretic approach. Baboon foraging decisions were influenced by multiple nonsocial and social factors and were often contingent on the characteristics of the habitat or individual. Differences in decision making between habitats were consistent with changes in interference-competition costs but not with changes in social-foraging benefits. Individual differences in decision making were suggestive of a trade-off between dominance rank and social capital. Our findings emphasize that taking a multifactor, multicontext approach is important to fully understand animal decision making. We also demonstrate how discrete-choice models can be used to achieve this.

Keywords: chacma baboons, habitat dependent, individual dependent, interference competition, multifactor, social foraging.

\section{Introduction}

How social foragers decide which patch to use is an important component of their foraging behavior. At any given decision point, an individual's patch preference may be influenced by many nonsocial and social factors, such as patch food content or the number of resident conspecifics, whose relative importance can vary, depending on the wider characteristics of the habitat and the forager itself (Giraldeau and Caraco 2000; Waite and Field 2007). However, the dif-

* Corresponding author; e-mail: harry.marshall04@ic.ac.uk.

Am. Nat. 2012. Vol. 180, pp. 481-495. (c) 2012 by The University of Chicago. 0003-0147/2012/18004-53644\$15.00. All rights reserved.

DOI: $10.1086 / 667587$ ferential influence of multiple factors depending on the habitat and forager's characteristics has yet to be fully explored. Most previous studies have either investigated the effects of a relatively wide range of factors, but not how their influence varies with habitat or individual characteristics (Di Bitetti and Janson 2001; Smith et al. 2001; Ha et al. 2003; Kazahari and Agetsuma 2008; King et al. 2011), or explored the influence of habitat and individual characteristics, but only on a narrow range of factors (Lendvai et al. 2004; Johnson et al. 2006; Sargeant et al. 2006; Kurvers et al. 2010; Metz et al. 2011).

Only two studies have attempted to consider the relative importance of a wide range of factors across different contexts (habitats or individuals) by adopting a multifactor, multicontext approach, and both these studies have suffered limitations. In the first case, Fortin and Fortin (2009) investigated how bison (Bison bison) resource-selection tactics varied with seasonal habitat changes. However, they were unable to identify individuals and so to examine either how foraging tactics varied between individuals with differing traits or how coforager traits influenced these tactics. In the second case, King et al.'s (2009) study of chacma baboon (Papio ursinus) foraging tactics was able to individually identify focal foragers and coforagers. However, this study considered only the effects of the nearest coforager on patch preference rather than the effects of all individuals in either the chosen patch or nearby alternative patches, and it did not investigate the effects of the patches' nonsocial characteristics.

The need to consider the many characteristics of multiple alternative patches, whose identity is likely to change between decisions, may have prohibited previous studies of foraging decisions from taking a multifactor, multicontext approach. Conventional statistical techniques used in ecology either do not allow the set of alternatives to change between decisions (e.g., resource-selection functions; Manly et al. 2002) or do not allow the consideration of more than two alternative patches (binomial models). Both these ap- 
proaches, however, are allowed by discrete-choice analysis, a technique developed in the study of human consumer choice (Ben-Akiva and Lerman 1985; Train 2003). Within ecology, discrete-choice analysis has been used in studies of broad-scale habitat use (reviewed in Cooper and Millspaugh 1999; Manly et al. 2002), but it has been used only twice in behavioral ecology, to consider cheetah (Acinonyx jubatus) hunting decisions (Cooper et al. 2007) and to investigate female greater prairie chicken (Tympanuchus $\mathrm{cu}$ pido) mate choice (Nooker and Sandercock 2008).

Discrete-choice analysis is designed to explore the factors influencing individual decision making, a topic that many studies in behavioral ecology aim to explore. An increased appreciation and application of this analytical tool may, therefore, lead to greater insight into animal decision making. In this article, we aim to demonstrate this potential by using it to explore the factors influencing foraging decisions in a wild population of social foragers, chacma baboons ( $P$. ursinus). We start by using it to identify the nonsocial and social influences on "baseline" patch preference, and then we show how it can be used to explore how the factors influencing patch preference differ between two habitats and between individuals with differing social traits.

\section{Methods}

\section{The Discrete-Choice Model}

The discrete-choice model assumes that, faced with a set of resources (the "choice set"), individuals seek to choose the resource that will give them the maximum utility (Cooper and Millspaugh 1999), where utility represents some measure of "satisfaction." Behavioral ecologists might think of utility as measuring the cost-benefit ratio of the different available resources, for example, in foraging decisions as the foraging reward of different food patches. In the model, the utility of the $i$ th resource in the $j$ th choice is defined as

$$
U_{i j}=\beta_{1} x_{i j 1}+\ldots+\beta_{n} x_{i j n}+\varepsilon_{i j}
$$

(Cooper and Millspaugh 1999). Here, $x_{i j}$ describe $n$ attributes of the resources and surrounding environment, $\beta$ represents the model parameters to be estimated for each of the $n$ attributes, and $\varepsilon_{\mathrm{ij}}$ is an error term. Knowing the utility of a single resource is, however, uninformative, since the probability of its being chosen will depend on the utility of all other resources in the choice set. This probability is described by the multinomial logit model, where $k$ is the number of alternatives in the choice set:

$$
P_{j}(i \mid k)=\frac{\exp \left(\beta_{1} x_{i j 1}+\ldots+\beta_{n} x_{i j n}\right)}{\sum_{1}^{k} \exp \left(\beta_{1} x_{k j 1}+\ldots+\beta_{n} x_{k j n}\right)}
$$

(McCracken et al. 1998; Cooper and Millspaugh 1999). This assumes that the error terms from equation (1) follow a
Type I extreme-value, or Gumbel, distribution (McCracken et al. 1998; Cooper and Millspaugh 1999).

Here, we demonstrate how the discrete-choice model can be used in the study of animal decision making by using it to analyze the foraging decisions made by wild baboons. In this context, the values of $i, k$, and $j$ in equations (1) and (2) represent a particular food patch (i) within a selection of patches (the choice set containing a total of $k$ patches) at a particular decision point $(j)$. Equation (1) then represents the utility value of the particular patch to the baboon making this foraging decision, and equation (2) describes the baboon's preference for the patch, given its characteristics and those of the surrounding patches.

\section{Study System}

Fieldwork was carried out at Tsaobis Leopard Park, Namibia $\left(22^{\circ} 23^{\prime} \mathrm{S}, 15^{\circ} 45^{\prime} \mathrm{E}\right)$, from June to November 2009. Data were collected from all adults in two troops of chacma baboons (19 and 12 adults in troops of 44 and 32, hereafter the "large" and "small" troops, respectively) on handheld Motorola MC35 Personal Digital Assistants using a customized spreadsheet in SpreadCE, version 2.03 (Bye Design 1999). Trained observers followed each study troop on foot from dawn to dusk. All baboons were habituated to the presence of human observers at close proximity and were individually recognizable using unique ear-notch combinations, made during previous capture events, and other naturally occurring distinguishing features (see Huchard et al. 2010 for further details). Observation conditions at Tsaobis are excellent, allowing detailed observations of individual- and group-level foraging behavior.

Baboons at Tsaobis forage predominantly in two habitats, woodland and desert. Both contain discrete food patches but differ markedly in their patch types and distribution. The woodland habitat's food patches are mainly large trees and bushes (median patch surface area of $156 \mathrm{~m}^{2}$, interquartile range $=28-237 \mathrm{~m}^{2}$; see below for sample sizes and calculations), such as Faidherbia albida, Prosopis glandulosa, and Salvadora persica, containing large numbers of food items (median of 87 items, interquartile range $=24-390$ items, such as pods, berries, flowers, and leaves). The surrounding desert habitat's food patches are mainly herbs and dwarf shrubs (median patch surface area of $0.4 \mathrm{~m}^{2}$, interquartile range $=0.4-3.5 \mathrm{~m}^{2}$ ), such as Monechma cleomoides, Sesamum capense, and Commiphora virgata, and they contain small numbers of food items (median of 9 items, interquartile range $=7-33$ items; see Cowlishaw and Davies 1997 for more details). Baboon troops move through their environment as a coherent and behaviorally synchronized group (King and Cowlishaw 2009), such that all individuals are typically foraging in the same habitat. 


\section{Data Collection}

Data were collected using focal follows (Altmann 1974) and monthly phenological surveys. Individual baboon foraging decisions were recorded using "patch-preference" focal follows. In addition, data on the size and food content of patches, and the social relationships between the focal forager and patch occupants, were collected separately using monthly phenological surveys and "social-behavior" focal follows, respectively. In both focal protocols, individuals were selected to ensure even sampling from all times of day, throughout the field season, and across all individuals. No individual was sampled more than once a day under each protocol. Two adult males emigrated from the large troop during the study and so were excluded from the analysis, leaving data from 29 adults (17 and 12 per troop).

Each patch-preference focal follow comprised one foraging decision-defined as the focal individual eating an item from a patch - and recorded the discrete choice set at this decision point. The choice set consisted of the chosen patch and all other patches within 5 and $20 \mathrm{~m}$ in the desert and woodland habitats (reflecting the differences in patch spacing between these habitats), respectively. Patches were defined as herbs, shrubs, or trees with no other conspecific plant within $1 \mathrm{~m}$ (closer conspecifics, which could potentially be reached by the forager without moving, were treated as part of the same patch). The patch species sampled represented the vast majority of the baboons' diet during the austral winter season. In each focal follow, the observer scanned the choice set as soon as the focal forager made a decision and recorded the characteristics of the patches within it. The nonsocial characteristics recorded were the food-item density, patch size, and food-item type. Fooditem density and patch size were scored on ordinal scales (app. A, available online), while food-item type was classified as having a handling time that was either low (flowers, leaves, and berries) or high (pods, bark, and roots). The social characteristics recorded were the number of occupants in each patch and the identity of any adult occupants. Overall, $24 \pm 5$ (mean \pm SD) choice sets were recorded for each focal individual (generating 703 choice sets in total), with a median of 7 patches (range $=1-21$ ) per choice set.

Patch size and food-item density scores were converted to patch quality (food content) and size $\left(\mathrm{m}^{2}\right)$ estimates using more-detailed patch information taken from monthly phenological surveys in the two habitats. In the desert, 132 food patches that fell within eight randomly placed $50 \times 1-\mathrm{m}$ transects within the study troops' home ranges were surveyed. In the woodland, 59 food patches, representative of patch species, size, and location, were selected from an earlier survey of 3,444 woodland patches (the Swakop River Survey; G. Cowlishaw, unpublished data). In each monthly survey, each patch's food-item density was scored as in the patch-preference focal follows (app. A), and the actual number of food items it contained was estimated. Each patch's size was also scored as in the patch-preference focal follows (app. A), and in the desert survey each patch's height, width, and depth in centimeters were also measured. The area and height of the woodland patches were already known, through differential GPS perimeter analysis and observer assessments, respectively, from the Swakop River Survey. The conversion of patch size and food-item density scores to patch quality and size estimates is detailed below.

Further behavioral data describing the social relationships between troop members were recorded using social-behavior focal follows. These lasted from 20 minutes to an hour (all follows lasting less than 20 minutes were discarded) and recorded the focal individual's grooming and aggressive interactions with others. In all cases, the direction of the interaction and the partner's identity were recorded, and for grooming, the interaction's duration was also recorded. Focal individuals were sampled in a stratified manner at all times of day throughout the field season to ensure representative social behavior from all habitats and activities. In total, $47.6 \pm 5.5$ hours (mean $\pm \mathrm{SD}$ ) of social-behavior focal follows were conducted for each of the 29 study animals. Aggressive interactions were also recorded ad lib.

\section{Data Processing}

The food content of patches recorded in the choice sets was predicted, using linear mixed-effect models, from the relationship between the food content (the response variable) and the ordinal patch size and food density scores (fixed effects) estimated from the monthly phenological survey data (app. A). Where possible, specific models for each food type and species combination (e.g., F. albida flowers) were calculated. However, where samples were too small, the data were pooled to create general food-item (e.g., all flowers) or species (e.g., all F. albida items) models. Patch ID was included as a random effect in all models, while food-item type was included in general patch-species models and species was included in general food-item models. In all models, empty patches were removed, and the response variable was $\log$ transformed to normalize the residuals (see table Al, available online).

To explore the physical constraints on forager space, it was necessary to estimate the size of patches on a continuous scale that would be comparable across all patches and habitats (the ordinal scoring systems for patch size in the choice sets were both species and habitat specific). To do this, data from the Swakop River Survey (for the woodland patches) and monthly phenological surveys (for the desert patches) were used to describe the relationship between the actual surface area of a patch (the response variable) and its ordinal size score (the explanatory variable) using linear models 
(app. A). Surface area was selected as a measure of patch size, because the majority of food items were found on the outer branches of plants, and was calculated from patch measurements (height, diameter) on the assumption that patches were cylindrical. Species-specific relationships were estimated for trees and bushes in the woodland, whereas the great diversity of desert herbs and shrubs meant that the number of each species in our survey was too small for species-specific models, so a general "herbs and shrubs" model was estimated (app. A). Surface area was log transformed to normalize the residuals in all models (see table A4, available online).

To explore the effects of a forager's social relationships on patch preferences, we calculated its mean rank difference, social capital, and relatedness to the adult occupants of each patch in the choice set. Each troop's adult dominance hierarchy was calculated from all dominance interactions recorded both during social-behavior focals and ad lib. (total number of interactions per troop: $n_{\text {large }}=1,655, n_{\text {small }}=$ 1,316) using Matman 1.1.4 (Noldus Information Technology 2003). In both troops, the hierarchy was strongly linear (Landau's corrected linearity index: $h_{\text {large }}^{\prime}=0.84, h_{\text {small }}^{\prime}=$ $1, P<.001$ in both). To control for troop size, each individual's rank was standardized to between 0 (most subordinate) and 1 (most dominant) as $1-[(1-r) /(1-n)]$, where $r$ is an individual's rank and $n$ is the number of individuals in the hierarchy.

Social animals maintain affiliative bonds with other group members through social interactions. In primates, these bonds are commonly measured by grooming interactions, and there is evidence that they may "trade" these interactions for commodities such as tolerance during foraging (Barrett et al. 1999; Fruteau et al. 2009). Social capital between foragers was therefore calculated using a grooming symmetry score (on the basis that a grooming asymmetry might indicate the "purchase" of tolerance during cofeeding): $\left[g_{i j} /\left(g_{i j}+r_{i j}\right)\right]-0.5$. Here, $g_{i j}$ is the proportion of focal $i$ s social-behavior focal time that it was observed grooming individual $j$ and $r_{i j}$ is the proportion of this time that individual $j$ groomed focal $i$. A score of -0.5 indicates that focal $i$ received all grooming interactions from $j$ (i.e., low social capital), +0.5 indicates $i$ contributed all grooming interactions (i.e., high social capital), and 0 indicates that both $i$ and $j$ contributed equally.

Pairwise relatedness $(r)$ between individuals was estimated using Wang's triadic estimator (Wang 2007) on the basis of 16 microsatellite loci. See Huchard et al. (2010) for further details.

\section{Analysis}

We analyzed baboon foraging decisions by using the discrete-choice model (see eqq. [1] and [2]). As there were multiple nonsocial and social factors that might influence patch preference, we used an information-theoretic approach to select estimates of the patch-preference model in equation (2) (Burnham and Anderson 2002; Garamszegi 2011). We generated a set of candidate models (table 1), assuming that foraging baboons were unable to consider the number of occupants in a patch without also considering its nonsocial characteristics and, similarly, unable to consider their relationship with the patch occupants without also considering their number. The relationship between group size and foraging reward is often shown to be humped: initially, the benefits of group foraging (such as the reduced need for antipredator vigilance and increased availability of social information) rise faster than the intraspecific-competition costs until, past a certain coforager number, this relationship reverses (Giraldeau and Caraco 2000; Krause and Ruxton 2002; Beauchamp 2007). Furthermore, the overall intensity of intraspecific competition, and so the particular form of this humped relationship, can be dependent on forager density (Rutten et al. 2010) and food-item handling times (Cresswell et al. 2001; Folmer et al. 2010). Therefore, we included a quadratic term for the number of patch occupants and terms for the interactions between the number of patch occupants and both the patch size and food-item handling time. Patch size was included as a controlling physical factor in all models including social predictors (models 3-66; table 1).

We then estimated the $\beta$ parameters in equation (2) in nine separate analyses. The first analysis explored baseline patterns of patch preference (across all habitats and individuals), the next two analyses explored differences in patch preference between two habitats (desert and woodland), and the final six analyses explored differences in patch preference between individuals with different social characteristics (involving three measures of social "advantage" and three of social "disadvantage"). Forager ID and habitat intercepts were included in all models to allow for unequal numbers of foraging decisions from each individual and habitat in the data sets, except in the habitat-specific analyses, where each model included only data from one habitat type and so only forager ID intercepts were included. The data sets used in each of these analyses were constructed as follows.

To produce a balanced data set, each choice set was randomly sampled without replacement to leave the chosen patch and three alternative patches. This resulted in 20 choice sets being discarded, leaving 683 sets in the full data set. Each row in this data set represented one patch and contained information as to whether it had been chosen $(0 /$ 1 ), the choice set it was from, and a range of explanatory variables describing its nonsocial and social characteristics (the $x$ 's in eqq. [1], [2]). To make comparisons between parameter estimates easier, each explanatory variable was standardized to have a mean of 0 and a standard deviation 
Table 1: Candidate models explaining patch preference in foraging baboons

\begin{tabular}{|c|c|c|}
\hline No. & Model & Notation \\
\hline None & Null & $\beta_{0}$ \\
\hline \multicolumn{3}{|c|}{ Nonsocial: } \\
\hline 1 & Food content & $\beta_{0}+\beta_{1}($ food $)$ \\
\hline 2 & Food + handling time & $\beta_{0}+\beta_{1}($ food $)+\beta_{2}($ handling $)$ \\
\hline \multicolumn{2}{|c|}{ Nonsocial + occupant number } & $\beta_{0}+\beta_{1}($ food $)+\beta_{2}$ (handling $)+\beta_{3}$ (size $)$ plus: \\
\hline 3 & Linear occupant effect & $\beta_{4}(\mathrm{occ})$ \\
\hline 4 & Nonlinear occupant effect & $\beta_{4}(\mathrm{occ})+\beta_{5}\left(\mathrm{occ}^{2}\right)$ \\
\hline 5 & Moderated by patch size & $\beta_{4}($ occ $)+\beta_{6}($ occ $\times$ size $)$ \\
\hline 6 & Moderated by patch size & $\beta_{4}(\mathrm{occ})+\beta_{5}\left(\mathrm{occ}^{2}\right)+\beta_{6}(\mathrm{occ} \times$ size $)+\beta_{7}\left(\mathrm{occ}^{2} \times\right.$ size $)$ \\
\hline 7 & Moderated by handling time & $\beta_{4}(\mathrm{occ})+\beta_{8}(\mathrm{occ} \times$ handling $)$ \\
\hline 8 & Moderated by handling time & $\begin{array}{l}\beta_{4}(\mathrm{occ})+\beta_{5}\left(\mathrm{occ}^{2}\right)+\beta_{8}(\mathrm{occ} \times \text { handling })+ \\
\beta_{9}\left(\mathrm{occ}^{2} \times \text { handling }\right)\end{array}$ \\
\hline 9 & Moderated by patch size and handling time & $\beta_{4}($ occ $)+\beta_{6}($ occ $\times$ size $)+\beta_{8}($ occ $\times$ handling $)$ \\
\hline 10 & Moderated by patch size and handling time & $\begin{array}{c}\beta_{4}(\text { occ })+\beta_{5}\left(\text { occ }^{2}\right)+\beta_{6}(\text { occ } \times \text { size })+\beta_{7}\left(\text { occ }^{2} \times \text { size }\right)+ \\
\beta_{8}(\text { occ } \times \text { handling })+\beta_{9}\left(\text { occ }^{2} \times \text { handling }\right)\end{array}$ \\
\hline \multicolumn{2}{|c|}{ Nonsocial + occupant number + relationship with occupants } & Models 3-10 plus: \\
\hline $11-18$ & Rank & $\beta_{10}($ rank $)$ \\
\hline $19-26$ & Grooming symmetry score & $\beta_{11}$ (symmetry) \\
\hline $27-34$ & Kinship & $\beta_{12}($ kinship $)$ \\
\hline $35-42$ & Rank + grooming symmetry & $\beta_{10}($ rank $)+\beta_{11}$ (symmetry $)$ \\
\hline $43-50$ & Rank + kinship & $\beta_{10}($ rank $)+\beta_{12}($ kinship $)$ \\
\hline $51-58$ & Grooming symmetry + kinship & $\beta_{11}($ symmetry $)+\beta_{12}($ kinship $)$ \\
\hline $59-66$ & Rank + rooming symmetry + kinship & $\beta_{10}($ rank $)+\beta_{11}$ (symmetry) $+\beta_{12}$ (kinship) \\
\hline
\end{tabular}

Note: food $=$ number of food items in a patch; handling $=$ food patch classed as requiring high $(1)$ or low $(0)$ food-item handling time; size $=$ patch surface area $\left(\mathrm{m}^{2}\right) ;$ occ $=$ number of individuals (occupants) in a food patch; rank = mean rank difference between the forager and all adult patch occupants; symmetry $=$ mean grooming symmetry score between the forager and all adult patch occupants; kinship = mean relatedness coefficient between the forager and all adult patch occupants.

of 1 , except for the categorical handling-time variable, which was dummy coded $(1=$ high, $0=$ low $)$. No explanatory variables were highly correlated in any data set (Pearson's correlation coefficient $r<|0.5|$ in all cases; see app. B, available online), except for the patch size and number-of-patchoccupants terms ( $r>0.5$ in five of nine data sets) and the linear and quadratic occupants terms $(r>0.75$ in all data sets). Each row also contained further information about the habitat in which each foraging decision occurred and the characteristics of the focal baboon.

For the analysis of the baseline patch preference, the full data set was used. For the analysis of habitat-specific patch preference, the choice sets were filtered according to whether the focal forager had been in the desert (number of choice sets $n=452)$ or woodland $(n=231)$. For the analysis of individual trait-specific patch preference, the choice sets were filtered according to whether the focal forager was classed as socially advantaged or socially disadvantaged. Foragers were classed as advantaged or disadvantaged if they scored higher or lower than the troop median for relative dominance rank (number of choice sets $n_{\text {high }}=335$ and $\left.n_{\text {low }}=328\right)$, social capital $\left(n_{\text {high }}=323, n_{\text {low }}=340\right)$, and number of close kin (relatedness coefficient $\geq 0.25$; $n_{\text {high }}=250, n_{\text {low }}=277$ ). Choice sets from individuals with scores equal to the troop median were excluded from that analysis. Individuals' classification on each social axis was independent of their classification on other axes; that is, a high-ranked individual was not more or less likely have high social capital or a high number of close kin (Cochran-Mantel-Haenszel three-way $\chi^{2}$ test, $\chi^{2}=0.057, \mathrm{df}=1, P=$ $.81)$.

For each of the nine analyses, we estimated the parameters in equation (2) for each candidate model, using a generalized linear model with a multinomial error structure and logit link. Following Burnham and Anderson (2002), in the baseline analysis $n / k>40$ (where $n$ is the number of choice sets and $k$ is the number of parameters in the maximal model), so Akaike's Information Criterion values were calculated for each candidate model, while in all other analyses $n / k<40$ and so $\mathrm{AIC}_{\mathrm{c}}$ (Akaike's Information Criterion corrected for small sample sizes) values were used. In all analyses, the overdispersion coefficient $\hat{c}$ was less than 1 . To identify final parameter values for each of the nine analyses, we used full model set averaging, because the maximum Akaike's model weight in each analysis was low (maximum of 0.30 in the baseline analysis). This procedure uses the estimates for all models in the model set to calculate global coefficient and standard error estimates for each predictor. 
Table 2: Final averaged model for baseline, habitat-specific, and individual trait-specific patch preference

\begin{tabular}{|c|c|c|c|c|c|c|c|c|c|c|c|c|c|c|}
\hline \multirow[b]{2}{*}{ Model } & \multicolumn{3}{|c|}{$\begin{array}{l}\text { Nonsocial patch } \\
\text { characteristics }\end{array}$} & \multicolumn{5}{|c|}{ Social patch characteristics } & \multicolumn{4}{|c|}{ Nonsocial $\times$ social } & \multicolumn{2}{|c|}{$\begin{array}{l}\text { Sample } \\
\text { sizes }\end{array}$} \\
\hline & Food & Handling & Size & Occ & $\mathrm{Occ}^{2}$ & Rank & Symmetry & Kinship & $\begin{array}{c}\text { Size } \times \\
\text { occ }\end{array}$ & $\begin{array}{l}\text { Size } \times \\
\text { occ }^{2}\end{array}$ & $\begin{array}{l}\text { Handling } \\
\times \text { occ }\end{array}$ & $\begin{array}{l}\text { Handling } \\
\times \text { occ }^{2}\end{array}$ & $n$ & $i$ \\
\hline Baseline & & & & & & & & & & & & & 683 & 29 \\
\hline$\beta$ & .18 & -.54 & -.34 & .83 & -.08 & -.16 & .01 & -.03 & -.004 & .001 & .77 & -.09 & & \\
\hline SE & .06 & .15 & .10 & .15 & .02 & .05 & .03 & .04 & .04 & .01 & .26 & .05 & & \\
\hline Desert & & & & & & & & & & & & & 452 & 29 \\
\hline$\beta$ & 3.97 & -.81 & -1.41 & 1.61 & .02 & -.64 & -.05 & .01 & 2.40 & .25 & .16 & -.06 & & \\
\hline SE & 1.96 & .26 & .71 & .61 & .32 & .17 & .07 & .06 & 1.32 & 1.10 & .62 & .61 & & \\
\hline Woodland & & & & & & & & & & & & & 231 & 29 \\
\hline$\beta$ & .24 & -.34 & -.33 & 1.15 & -.12 & -.03 & .01 & -.03 & .00 & .001 & .26 & -.03 & & \\
\hline SE & .08 & .27 & .11 & .21 & .03 & .05 & .04 & .05 & .04 & .01 & .33 & .05 & & \\
\hline High rank & & & & & & & & & & & & & 335 & 14 \\
\hline$\beta$ & .19 & -.62 & -.28 & .96 & -.10 & -.07 & -.03 & -.02 & -.01 & .01 & .75 & -.10 & & \\
\hline SE & .10 & .22 & .14 & .24 & .04 & .07 & .05 & .04 & .06 & .01 & .38 & .06 & & \\
\hline Low rank & & & & & & & & & & & & & 328 & 14 \\
\hline$\beta$ & .22 & -.71 & -.43 & .97 & -.09 & -.08 & .19 & -.01 & .01 & -.002 & .07 & .00 & & \\
\hline SE & .09 & .21 & .15 & .24 & .03 & .09 & .08 & .05 & .06 & .01 & .21 & .03 & & \\
\hline High grooming symmetry & & & & & & & & & & & & & 323 & 14 \\
\hline$\beta$ & .10 & -.52 & -.10 & .65 & -.04 & -.15 & .02 & -.01 & .00 & .00 & .62 & -.10 & & \\
\hline SE & .08 & .22 & .13 & .23 & .04 & .08 & .05 & .04 & .05 & .01 & .43 & .11 & & \\
\hline Low grooming symmetry & & & & & & & & & & & & & 340 & 14 \\
\hline$\beta$ & .28 & -.56 & -.56 & 1.09 & -.12 & -.18 & .01 & -.04 & -.03 & .01 & .67 & -.05 & & \\
\hline SE & .10 & .22 & .15 & .24 & .04 & .08 & .04 & .06 & .08 & .02 & .40 & .07 & & \\
\hline High number of kin & & & & & & & & & & & & & 250 & 11 \\
\hline$\beta$ & .87 & -.78 & -.35 & 1.30 & -.36 & -.15 & .001 & .02 & 11 & -.03 & .04 & .03 & & \\
\hline SE & .31 & .26 & .18 & .44 & .14 & .11 & .05 & .05 & .20 & .06 & .39 & .16 & & \\
\hline Low number of kin & & & & & & & & & & & & & 277 & 12 \\
\hline$\beta$ & .10 & -.26 & -.28 & .69 & -.04 & -.16 & -.05 & -.15 & .06 & -.01 & .23 & -.03 & & \\
\hline SE & .08 & .23 & .17 & .31 & .04 & .08 & .07 & .08 & .11 & .02 & .36 & .06 & & \\
\hline
\end{tabular}

Note: Coefficient estimates and unconditional standard errors of estimates $(\beta \pm \mathrm{SE})$ are given for each explanatory variable; see table 1 for definitions of variable notations. $n$ foraging decisions from $i$ individuals were used to estimate each model. All explanatory variables, except the categorical handling-time variable, were standardized to a mean of 0 and a standard deviation of 1.

Global coefficient estimates are calculated as the sum of a predictor's coefficient estimates in all models, each weighted by the model's Akaike's weight. Thus, predictors that have stronger effects in better models (those with higher Akaike's weights) have a stronger effect in the final averaged model compared to those that appear or have a strong effect only in poorer models (those with lower Akaike's weights; Lukacs et al. 2010; Symonds and Moussalli 2011). Global standard error estimates were calculated using the unconditional estimator recommended by Lukacs et al. (2010).

We explored the predicted effects of specific patch characteristics ( $x$ 's) by calculating a patch's "selection index," defined by the numerator of equation (2), $\exp \left(\beta_{1} x_{i j 1}+\right.$ $\ldots+\beta_{n} x_{i j n}$ ) (Manly et al. 2002), over the observed range of $x$, keeping all other characteristics constant and using the back-transformed parameter estimates in the final averaged models. In figures 1 and 3, plots of these calculations are presented. However, in figure 2, it was necessary to present plots of the beta coefficients and patch utility (the natural logarithm of the selection index; see eq. [1]) to make comparisons between the desert and woodlands models easier. All analyses were carried out in R, version 2.12.1, using the lme4 package for mixed-model estimation and the mlogit package, version 0.1-8, for multinomial logit model estimation (Croissant 2010; R Development Core Team 2010; Bates et al. 2011).

\section{Results}

In the baseline analyses (table 2; the 95\% confidence model set is available in Dryad: http://dx.doi.org/10.5061/ dryad.8m405), the final averaged model showed that both nonsocial and social factors strongly affected the baseline patch preference (fig. 1). The relative probability of a patch being chosen increased by $20 \%$ for every standard deviation (SD) increase in food content but declined by $42 \%$ if a patch had high handling-time requirements (fig. 1A). Patch size also had a negative effect, reducing the probability of a patch being chosen by $30 \%$ for every $1-\mathrm{SD}$ increase in size. The final baseline model also showed an important effect of some, but not all, candidate social factors. The number and rank of patch occupants had strong effects: patch preference peaked at intermediate numbers of occupants (5-6 individuals), but the peak height was moderated by the mean rank difference between the occupants and the forager (fig. 1B). Thus, in- 


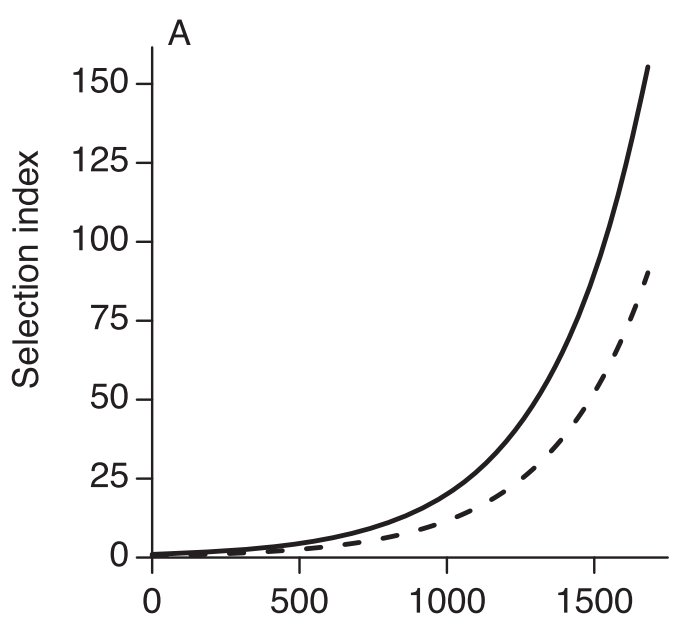

Food content (00s food items)



Number of patch occupants



Number of patch occupants dependent of occupant number, a midranking forager was 4.1 times more likely to choose a patch containing the lowest-ranking occupant over a patch containing the highest-ranking occupant. In contrast, the effects of mean social capital or the mean relatedness between the forager and patch occupants were weak. Finally, the interactive effect of occupant number and food handling time was also important. Patches with food requiring low handling times were preferred when they contained high $(>7)$ or low $(\leq 2)$ numbers of occupants. However, at intermediate numbers of occupants, preference was stronger for foods with high handling times, such that when patches contained five occupants, a high-handling-time patch was $63 \%$ more likely to be chosen (fig. 1C). The effect of the interaction between patch size and number of occupants was weak.

In the woodland and desert analyses (table 2; the 95\% confidence model sets are available in Dryad: http:// dx.doi.org/10.5061/dryad.8m405), there were marked differences in the important factors influencing patch preference in each habitat's final averaged model (fig. 2). Nonsocial factors had a much stronger effect on patch preference in the desert habitat: an increase of $1 \mathrm{SD}$ in food content (fig. $2 A$ ), a high handling time, or a 1-SD increase in patch size (fig. $2 B$ ) made a patch 41.3 times more likely and 1.6 and 2.9 times less likely to be chosen in the desert than in the woodlands, respectively. The mean rank difference between foragers and patch occupants was also more influential in the desert (fig. 2B). There, a midranking individual was 349 times more likely to choose a patch containing the lowest-ranking occupant over one containing the highest-ranking one but, given the same foraging decision, was only 1.3 times more likely to do so in the woodlands. Finally, the interaction between patch size and number of occupants was highly important in the desert but negligible in the woodlands, where patch size had a moderately negative effect on patch preference independent of the number of patch occupants (fig. 2C). Foragers in the desert were 11.9 times more likely to choose the smallest over the largest patch when both were unoccupied, but this relationship reversed when the patches contained two occupants. There was some indi-

Figure 1: Baseline patch-preference model. $A$ shows the effect of patch food content on patch preference (as measured by the selection index) where patches have a high (dashed line) or low (solid line) handling time. $B$ shows the effect of number of patch occupants on patch preference where the mean rank difference between the forager and the patch occupants is -1 (forager subordinate; dotted line), 0 (solid line), and 1 (forager dominant; dashed line). $C$ shows the interactive effect between number of occupants and food handling time on patch selection index (solid line and circles for high handling time, dashed line and triangles for low handling time). 

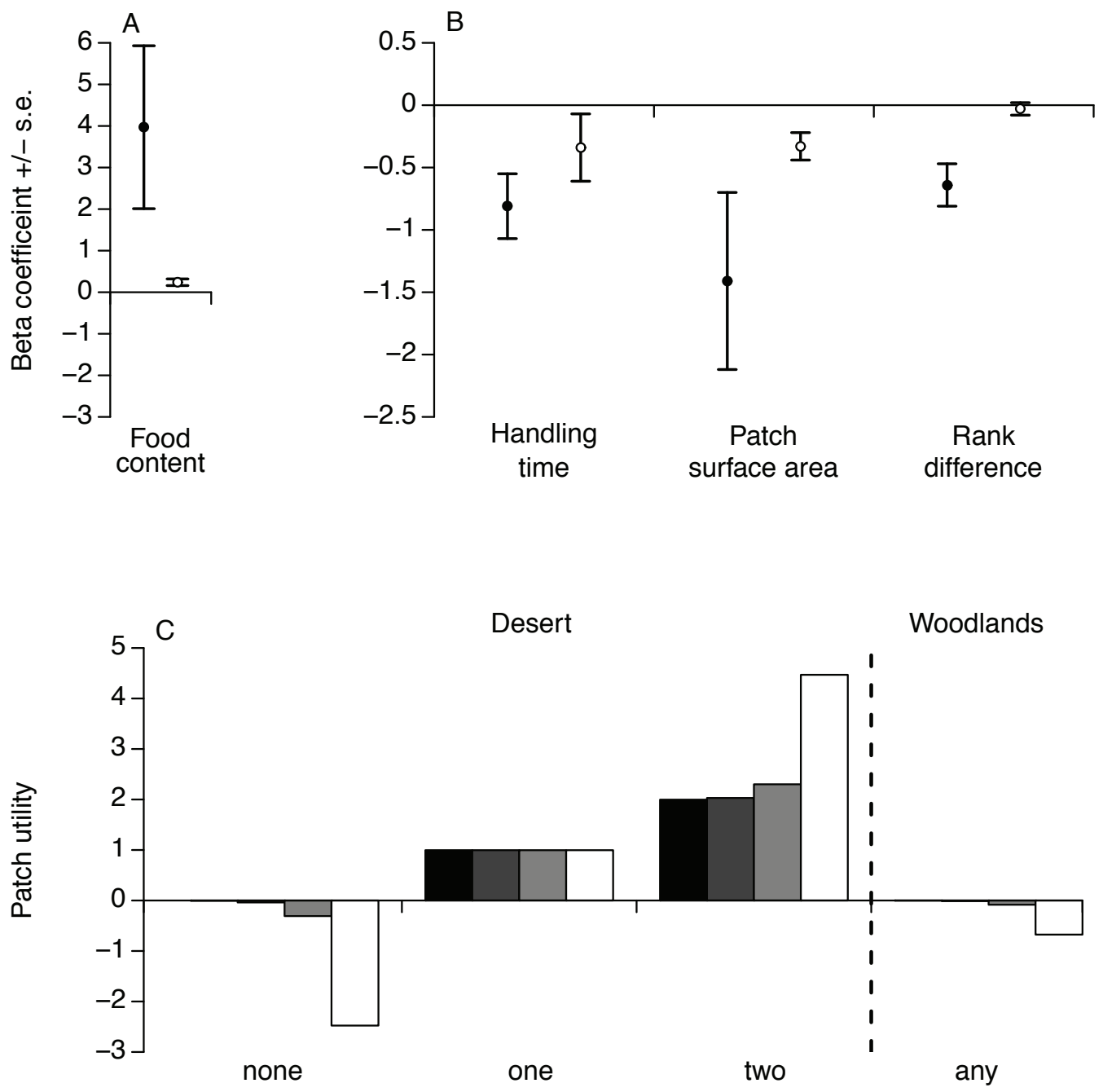

Number of patch occupants

Figure 2: Comparing the desert and woodlands habitats' patch-preference models. A compares the estimated beta coefficient ( \pm unconditional standard error $[\mathrm{SE}]$ ) in equation (2) for patch food content in the desert (filled circles) and woodlands (open circles) patchpreference models. $B$ makes the same comparison for food-item handling time, patch size (as measured by surface area), and the mean rank difference between a forager and patch occupants. $C$ shows the interactive effect of the number of occupants and patch size on patch utility (the natural logarithm of a patch's selection index; see eq. [1]) in the desert model and compares this to the effect of patch size on patch preference in the woodlands model, where its effect is independent of the number of patch occupants. The bars, left to right in each group, represent the four patch sizes estimated in the desert: 0.4, 3.5, 28.1, and $225 \mathrm{~m}^{2}$ (see app. A for scoring details).

cation that the influence of the number of occupants was a little stronger in the desert; however, the errors associated with the linear and quadratic effects in the desert model were relatively large. A forager's mean social capital with and mean relatedness to patch occupants had no effect in either habitat, nor did the interaction between the number of patch occupants and food-item handling time.
In the analyses of socially advantaged or disadvantaged foragers (table 2; the 95\% confidence model sets are available in Dryad: http://dx.doi.org/10.5061/dryad.8m405), the final averaged models showed noticeable differences in the factors influencing these foragers' decisions (fig. 3). The interaction between number of patch occupants and food-item handling time had a strong effect in high-ranked 

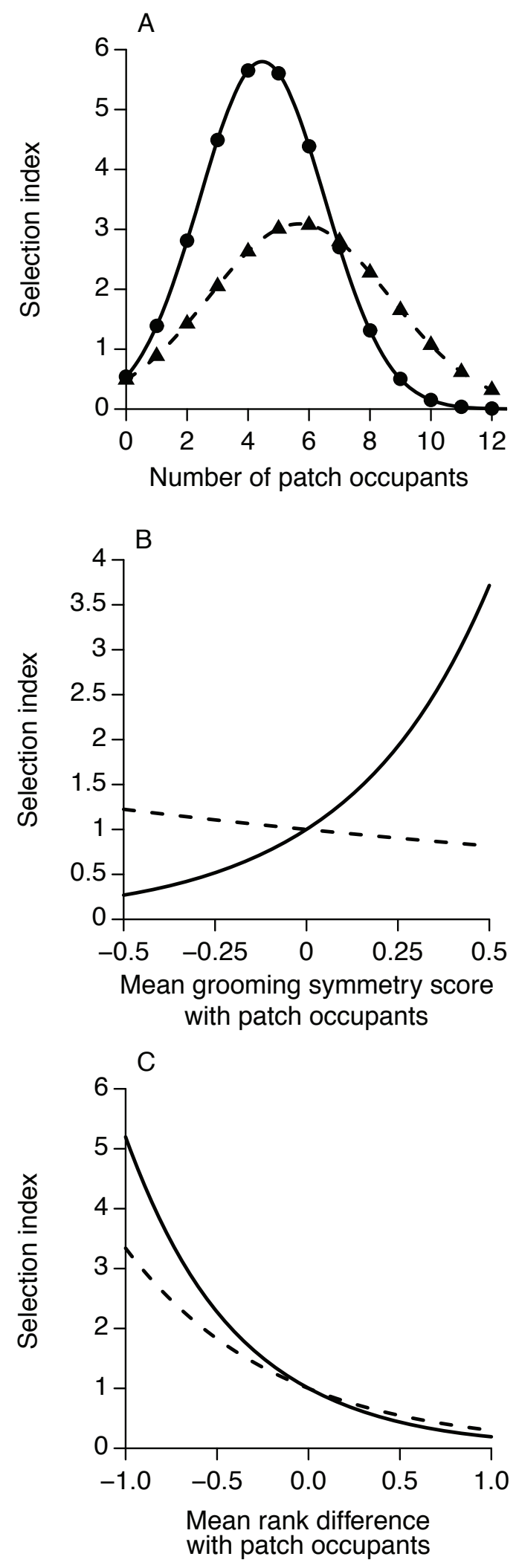

foragers but a negligible effect in low-ranked foragers, meaning that a high-handling-time patch containing five occupants was $86 \%$ more likely to be chosen by highrather than by low-ranked foragers (fig. 3A). Instead, lowranking foragers showed a much stronger effect of their mean social capital with patch occupants: they were 4.5 times more likely than high-ranked foragers to choose a patch containing individuals with whom they had contributed all grooming interactions and so held a high amount of social capital (symmetry score $=0.5$; fig. $3 B$ ). Similarly, the mean rank difference with patch occupants had a marginally stronger effect in foragers with low social capital: they were $18 \%$ more likely than foragers with high social capital to choose a patch containing the lowestranked occupant (fig. 3C). Mean kinship with patch occupants had a negligible effect on patch preferences when foragers were grouped by either rank or social capital. In contrast, when the patch preferences of individuals with many close kin in the social group were compared with those of individuals with few, there was no difference in the effect of the mean rank difference or mean social capital with patch occupants, but there was some indication of a difference in the effect of kinship: individuals with more close kin in the group showed a weak preference for patches containing such individuals, while individuals with fewer close kin in the group showed a strong aversion. Finally, there were also some differences in the effects of nonsocial factors and number of patch occupants. Patch preference in individuals with low social capital and a high number of kin in the group showed a greater effect of food content and patch size and potentially increased faster at lower occupant numbers and declined faster at higher occupant numbers (as described by the greater effects of the linear and quadratic occupant variables), compared to individuals with high social capital and a low number of close kin. No such differences occurred between individuals of high and low rank. The interactive effect of patch occupant number and patch size was similar across all models of socially advantaged or disadvantaged foragers.

\section{Discussion}

Our findings demonstrate how discrete-choice models can be used to explore the variable influences of multiple fac-

Figure 3: Comparing individual trait-specific patch-preference models. A shows the effect of occupant number on the preference for high-handling-time patches in high- (solid line) and low-ranked (dashed line) individuals. $B$ shows the effect of social capital (as measured by a forager's mean grooming symmetry score with patch occupants) on patch preference in low- (solid line) and high- (dashed line) ranked foragers. $C$ shows the effect of the mean rank difference between a forager and patch occupants on patch preference in foragers in with low (solid line) and high (dashed line) social capital. 
tors in multiple contexts on animal decision making. Each context-specific model allowed us to explore and compare the influence of multiple nonsocial and social factors on baboon foraging decisions. Using discrete-choice models in a multicontext framework further allowed us to show that the influence of these factors is often contingent on the habitat's characteristics and the forager's social traits. Previous work has shown that the drivers of foraging decisions can be numerous (Smith et al. 2001; Kazahari and Agetsuma 2008) and can vary between habitats (e.g., Johnson et al. 2006; Sargeant et al. 2006) and individuals (e.g., Lendvai et al. 2004; Kurvers et al. 2010). However, our study demonstrates that a multifactor, multicontext approach is likely to be invaluable in fully understanding and integrating the factors influencing animal decision making within a particular system and in identifying common trends across systems. Below, we discuss the use of discretechoice analysis in this approach and then the implications of the patterns of baboon foraging decisions in our analyses.

\section{Using Discrete-Choice Models to Study Animal Decision Making}

Our findings demonstrate how discrete-choice models can be used to take a multifactor, multicontext approach to the study of animal decision making. Their primary advantage is that they allow the influence of multiple factors on animal decisions to be quantified within a single analytical model. Unlike other multivariate methods, they allow two key elements of realism: multiple alternatives at each decision, rather than the two alternatives in binomial models, and changes in the identity of these alternatives between decisions, as opposed to the constant alternatives in classic resource-selection functions (Manly et al. 2002). These two elements are likely to be closer approximations of realism not only in the foraging behavior explored here but also in many other behaviors, such as mate choice and fighting decisions. In addition, discrete-choice models explicitly study the decision itself, in this example which patch the baboon used, rather than attempting to deduce it from an outcome such as the foraging success of the baboon within the patch. There is a growing appreciation that an animal's decisions are not always optimal, either because of incorrect decisions by the animal (Giraldeau et al. 2002; Houston et al. 2007) or because our understanding of what is optimal is flawed (Dall et al. 2004; Freidin and Kacelnik 2011). Using discrete-choice models to explicitly study the factors influencing decisions and comparing these to the factors determining the outcomes of these decisions may, therefore, provide new insights into how good animals are at making decisions, how good our understanding of optimality is, and how these vary between habitats and individuals.

There are, however, some limitations to the use of discrete-choice models. As a multivariate method, they are at risk of overparameterization, especially as researchers ask increasingly detailed questions requiring the inclusion of multiple explanatory variables. For example, in our baboon foraging analysis, the maximal model in each context required the estimation of 13 parameters (12 explanatory variables and the intercept). Ideally, rather than fitting one model per context, we would have simply fitted one model including interaction terms to explore how the factors influencing foraging decisions varied between the two habitats and between individuals' social traits. However, this led to a maximal model with 109 parameters (108 explanatory variables and the intercept) and convergence issues, even though we had a relatively large data set $(n=683)$. Discrete-choice models also require researchers to define what resources are "available" or, in this case, what patches the baboon could perceive. As in most other habitat-use analyses, this is dependent on researchers' judgment of what is biologically reasonable and so could leave the method open to being criticized as subjective. Previous studies using discrete-choice models (Cooper and Millspaugh 1999; Cooper et al. 2007) and other habitatuse analyses (Manly et al. 2002; McLoughlin et al. 2006) have been able to make this judgment and provide valuable insights into animal behavior and habitat use. Nevertheless, the development of a rigorous and standardized method for determining what makes a resource available would benefit this method in the future.

\section{Baboon Foraging Decisions}

Baboon foraging decisions were influenced by multiple nonsocial and social patch characteristics. Our baseline results were consistent with those of previous studies showing that increased food-handling time can reduce a forager's intake rate (Cresswell et al. 2001; Illius et al. 2002) and how, in social species, foraging success is also commonly characterized as having a humped relationship with foraging-group size (Krause and Ruxton 2002; Beauchamp 2007; MacNulty et al. 2011) and being influenced by coforagers' rank (Milinski and Parker 1991). Previous studies have also linked smaller patch size to increased interindividual aggression (Sirot 2000; Johnson et al. 2004). Our finding of a preference for smaller patches suggests that, in some cases, increased aggression costs in smaller patches can be outweighed by the improved foraging efficiency of searching for food in a smaller area (as our models controlled for the amount of food).

Previous studies have suggested that high handling time increases interference competition (Sirot 2000; Cresswell 
et al. 2001; Folmer et al. 2010), predicting that, across the humped relationship between patch preference and occupant number that we observed, foragers should show a lower preference for patches with high handling time. However, while we found this at low and high occupant numbers, we found the opposite at intermediate numbers. Food stealing, that is, kleptoparasitism, is common in many social-foraging species (Giraldeau and Dubois 2008), including baboons (Cheney and Seyfarth 2008; King et al. 2009; H. H. Marshall, personal observations), and it provides a potential tactic for foragers to avoid high handling times by stealing ready-processed food from subordinate animals. Our results, therefore, suggest that kleptoparasitism makes high-handling-time patches more profitable at intermediate numbers of patch occupants but that at lower numbers kleptoparasitism opportunities are too scarce and at higher numbers its benefits are outweighed by feeding-competition costs (Stillman et al. 1996; Sirot 2000). Beauchamp's (2008) spatial producer-scrounger model shows how a similar mechanism can produce a similar relationship between forager density and the use of the scrounging tactic (joining another forager on a patch but not necessarily stealing food from them). Scrounging initially increased with forager density, as opportunities to scrounge became more common, but then leveled off or decreased at higher forager densities as the number of foragers exploiting each food discovery increased, decreasing each scrounger's share. This model and our results suggest that the shape of the relationship between foraging-group size and individual forager success can be influenced by the opportunities for joining others' food discovery (scrounging) or actively stealing food from others (kleptoparasitism) that the underlying physical environment presents.

In addition to our exploration of how multiple factors can influence foraging decisions, our comparisons between different habitats and individuals have highlighted the importance of considering multiple contexts when studying animal decision making. When the desert and woodland habitat models were compared, the effect of all nonsocial patch characteristics was weaker in the relatively closed, low-visibility woodland. This supports previous studies' findings that a habitat's characteristics can constrain animals' ability to visually assess a patch's nonsocial characteristics (Cresswell et al. 2001; Fortin and Fortin 2009). More interestingly, this comparison also suggested that differences in the costs of social foraging between habitats may alter foraging decisions. The stronger negative effects of food-item handling time and coforager rank in the desert are consistent with more intense interference competition (Milinski and Parker 1991; Sirot 2000; Folmer et al. 2010). This suggestion is further supported by the interactive effect of patch size and number of occupants in the desert. There, the smallest patches were preferred when unoccupied, but when patches contained more than two occupants the largest were preferred, suggesting strongly density-dependent interference competition (e.g., Rutten et al. 2010). There was no suggestion, however, that the benefits of social foraging similarly varied between the two habitats. Previous work has suggested that predation risk (Cowlishaw 1997) and the benefits of social information use (Valone and Templeton 2002; Dall et al. 2005) can be habitat dependent. Yet if this were the case, we might have expected to see a habitat difference in the baboons' preferences for joining coforagers in a food patch, given that both safety from predators and the availability of social information increase with coforager number (Quinn and Cresswell 2004; King and Cowlishaw 2007). The fact that we did not may suggest that the proposed difference in interference competition between habitats overwhelmed any detectable variation in social-foraging benefits. Indeed, previous experimental studies reporting habitat-specific changes in the benefits of social foraging explicitly controlled for variation in interference competition (Giraldeau et al. 1994; Templeton and Giraldeau 1995; Rieucau and Giraldeau 2011). This highlights the importance of considering how behavior varies between natural, as well as between artificial or controlled, habitats. It also suggests that, in some cases, habitat-level variation in social-foraging benefits may be relatively unimportant compared to the costs.

The factors influencing foraging decisions also varied with individuals' social traits, their rank in particular. Previous studies have shown that subordinate foragers can adjust their behavior to maintain foraging gains in the face of dominant coforagers (Bugnyar and Kotrschal 2004; Hewitson et al. 2007; Held et al. 2010). We found support for this mechanism, showing that high-ranked individuals preferred high-handling-time patches at intermediate occupant numbers, presumably because of the greater number of kleptoparasitic opportunities (Stillman et al. 1996; Sirot 2000; Cresswell et al. 2001). In contrast, low-ranking individuals preferred patches containing coforagers with whom they had more social capital (higher grooming symmetry scores) and who were thus more likely to tolerate them, allowing greater foraging success (Fruteau et al. 2009; Tiddi et al. 2011). Silk et al. (2010b) suggested that the fitness costs imposed by low rank in baboons were offset by improved social relationships, and our findings seem to indicate one possible mechanism through which this offsetting might occur. There was also some suggestion of a complementary effect, since individuals with low social capital had a somewhat stronger preference for patches containing lower-ranked occupants than did individuals with high social capital. This difference was not, however, as marked as that between high- and low-ranked individ- 
uals' use of social capital. This may reflect the fact that while low-ranked individuals can compensate for their social position by investing more in social capital outside of foraging periods, comparable opportunities for individuals with low social capital to invest in rank do not exist, since rank is not a tradeable commodity (indeed, female rank is maternally inherited, while male rank depends on competitive ability). To similarly compensate for their social disadvantage, individuals with low social capital might therefore be expected to place greater emphasis on patches' nonsocial characteristics and the number of coforagers present when making foraging decisions, and this is exactly what we observed. Studies on the influence of social relationships on social-foraging performance (Barrett et al. 1999; Fruteau et al. 2009) and individual fitness (Silk 2007) have tended to focus on primates. However, there is emerging evidence that they can have positive effects on foraging performance (Beauchamp 2000; Carter et al. 2009) and fitness (Cameron et al. 2009; Barocas et al. 2011) in other social systems. Our suggested mechanism by which foragers offset fitness costs of low rank by using their social relationships may, therefore, be found in other primate and nonprimate social systems.

Differences between foraging decisions made by individuals with high versus low numbers of close kin were less clear. In general, the effect of kinship on social-foraging behavior in previous studies has been mixed (Ha et al. 2003; Tóth et al. 2009; Mathot and Giraldeau 2010). Mathot and Giraldeau (2010) suggest that one explanation for these mixed results may be that, while foragers outside a patch are expected to avoid imposing coforaging costs on kin by joining patches containing non-kin, patch occupants are expected to prefer foragers who are kin joining the patch. They modeled both of these considerations within a producer-scrounger framework and predicted greater scrounging among kin. In support of this model, there was some evidence that individuals' preference for joining patches containing kin increased with the number of close kin they had in the troop. However, this model would also suggest that high-ranked individuals, who have greater control over patch entry, should show a greater preference for avoiding kin, which they do not. There are two reasons we may have failed to find a strong effect of kinship on foraging decisions in this study. First, the effects of kinship on foraging baboons and other social primates may be low, as there is evidence that daily access to food is negotiated primarily through social capital (Barrett et al. 1999; Fruteau et al. 2009; Henzi et al. 2009), while kinship provides longer-term benefits, such as social support (Silk et al. 2010a) and reduced stress levels (Crockford et al. 2008). Second, like previous studies that failed to find a clear kinship effect on foraging at this site (King et al. 2009, 2011), we considered only adult patch occupants, who have relatively low levels of relatedness compared to adults and juveniles (e.g., parent-offspring vs. half-sibling relationships). To understand the role of kinship in social foraging, therefore, it may be important to consider all social-group members.

In overview, multiple factors influence baboons' foraging decisions, and the influence of these factors varies between habitats and between individuals with differing social traits. This variation is consistent with baboons adjusting their decision making in response to the differing foraging costs and benefits associated with these habitat and individual contexts. Many of these factors and contexts have been shown to influence social-foraging behavior in a similar manner across a broad range of socialforaging systems, including birds (e.g., Johnson et al. 2006; Folmer et al. 2010), ungulates (Fortin and Fortin 2009; Held et al. 2010), carnivores (Waite and Field 2007; Metz et al. 2011), and cetaceans (Sargeant et al. 2006). This suggests that our finding that baboon foraging decisions are influenced by multiple factors in a context-dependent fashion is unlikely to be exceptional. Instead, multifactor, multicontext systems of social-forager decision making are likely to be widespread. Describing these systems will be important in furthering our understanding of socialforaging behavior. As we have demonstrated here, discretechoice models can provide a powerful tool to further this investigation.

\section{Acknowledgments}

Thanks to W. Birkin, R. Bodenham, K. Forsythe, J. Iles, H. Peck, C. Sick, and W. Symes for assistance with data collection in the field. Permission to work at the field site was kindly granted by the Ministry of Lands and Resettlement (Tsaobis Leopard Park) and the Snyman and Wittreich families (surrounding farms). We also thank the Gobabeb Training and Research Centre for affiliation and the Ministry of Environment and Tourism for research permission in Namibia. The manuscript was greatly improved by comments from the Institute of Zoology's Behaviour Discussion Group, I. Owens, I. Smallegange, and three anonymous reviewers. We confirm that we adhered to the Guidelines for the Use of Animal Behaviour for Research and Teaching (Animal Behaviour 2003, 65:249255 ) and legal requirements of the country (Namibia) in which fieldwork was carried out. H.H.M. was supported by a Natural Environment Research Council Open CASE (Collaborative Award in Science and Engineering) studentship (NE/F013442/1) with the Zoological Society of London (ZSL) as CASE partner. A.J.C. was supported by a Fenner School of Environment and Society studentship and grants from the Leakey Foundation, the Animal Be- 
havior Society (USA), the International Primatological Society, and the Explorers Club Exploration Fund. This article is a publication of the ZSL Institute of Zoology's Tsaobis Baboon Project.

\section{Literature Cited}

Altmann, J. 1974. Observational study of behavior: sampling methods. Behaviour 49:227-267.

Barocas, A., A. Ilany, L. Koren, M. Kam, and E. Geffen. 2011. Variance in centrality within rock hyrax social networks predicts adult longevity. PLoS ONE 6:e22375.

Barrett, L., S. P. Henzi, T. Weingrill, J. Lycett, and R. Hill. 1999. Market forces predict grooming reciprocity in female baboons. Proceedings of the Royal Society B: Biological Sciences 266:665670.

Bates, D., M. Maechler, and B. Bolker. 2011. lme4: linear mixedeffects models using S4 classes. R package. Version 0.999375-39. http://cran.r-project.org/web/packages/lme4//index.html.

Beauchamp, G. 2000. The effect of prior residence and pair bond on scrounging choices in flocks of zebra finches, Taenopygia guttata. Behavioural Processes 52:131-140.

2007. Effect of group size on feeding rate when patches are exhaustible. Ethology 113:57-61.

- 2008. A spatial model of producing and scrounging. Animal Behaviour 76:1935-1942.

Ben-Akiva, M., and S. Lerman. 1985. Discrete choice analysis: theory and application to travel demand. MIT Press, Cambridge, MA.

Bugnyar, T., and K. Kotrschal. 2004. Leading a conspecific away from food in ravens (Corvus corax)? Animal Cognition 7:69-76.

Burnham, K. P., and D. R. Anderson. 2002. Model selection and multimodel inference: a practical information-theoretic approach. 2nd ed. Springer, New York.

Bye Design. 1999. SpreadCE. Version 2.03. www.byedesign.co.uk.

Cameron, E. Z., T. H. Setsaas, and W. L. Linklater. 2009. Social bonds between unrelated females increase reproductive success in feral horses. Proceedings of the National Academy of Sciences of the USA 106:13850-13853.

Carter, A. J., S. L. MacDonald, V. A. Thomson, and A. W. Goldizen. 2009. Structured association patterns and their energetic benefits in female eastern grey kangaroos, Macropus giganteus. Animal Behaviour 77:839-846.

Cheney, D. L., and R. M. Seyfarth. 2008. Baboon metaphysics: the evolution of a social mind. University of Chicago Press, Chicago.

Cooper, A. B., and J. J. Millspaugh. 1999. The application of discrete choice models to wildlife resource selection studies. Ecology 80: 566-575.

Cooper, A. B., N. Pettorelli, and S. M. Durant. 2007. Large carnivore menus: factors affecting hunting decisions by cheetahs in the Serengeti. Animal Behaviour 73:651-659.

Cowlishaw, G. 1997. Trade-offs between foraging and predation risk determine habitat use in a desert baboon population. Animal Behaviour 53:667-686.

Cowlishaw, G., and J. Davies. 1997. Flora of the Pro-Namib Desert Swakop River catchment, Namibia: community classification and implications for desert vegetation sampling. Journal of Arid Environments 36:271-290.

Cresswell, W., R. D. Smith, and G. D. Ruxton. 2001. Absolute foraging rate and susceptibility to interference competition in blackbirds varies with patch conditions. Journal of Animal Ecology 70:228236.

Crockford, C., R. Wittig, P. L. Whitten, R. M. Seyfarth, and D. L. Cheney. 2008. Social stressors and coping mechanisms in wild female baboons (Papio hamadryas ursinus). Hormones and Behavior 53:254-265.

Croissant, Y. 2010. mlogit: multinomial logit model. R package. Version 0.1-8. http://cran.r-project.org/web/packages/mlogit/.

Dall, S. R. X., L. A. Giraldeau, O. Olsson, J. M. McNamara, and D. W. Stephens. 2005. Information and its use by animals in evolutionary ecology. Trends in Ecology \& Evolution 20:187-193.

Dall, S. R. X., A. I. Houston, and J. M. McNamara. 2004. The behavioural ecology of personality: consistent individual differences from an adaptive perspective. Ecology Letters 7:734-739.

Di Bitetti, M., and C. Janson. 2001. Social foraging and the finder's share in capuchin monkeys. Animal Behaviour 62:47-56.

Folmer, E. O., H. Olff, and T. Piersma. 2010. How well do food distributions predict spatial distributions of shorebirds with different degrees of self-organization? Journal of Animal Ecology 79: 747-756.

Fortin, D., and M.-E. Fortin. 2009. Group-size-dependent association between food profitability, predation risk and distribution of freeranging bison. Animal Behaviour 78:887-892.

Freidin, E., and A. Kacelnik. 2011. Rational choice, context dependence, and the value of information in European starlings (Sturnus vulgaris). Science 334:1000-1002.

Fruteau, C., B. Voelkl, E. van Damme, and R. Noë. 2009. Supply and demand determine the market value of food providers in wild vervet monkeys. Proceedings of the National Academy of Sciences of the USA 106:12007-12012.

Garamszegi, L. Z. 2011. Information-theoretic approaches to statistical analysis in behavioural ecology: an introduction. Behavioral Ecology and Sociobiology 65:1-11.

Giraldeau, L.-A., and T. Caraco. 2000. Social foraging theory. Princeton University Press, Princeton, NJ.

Giraldeau, L.-A., and F. Dubois. 2008. Social foraging and the study of exploitative behavior. Advances in the Study of Behavior 38: 59-104.

Giraldeau, L.-A., C. Soos, and G. Beauchamp. 1994. A test of the producer-scrounger foraging game in captive flocks of spice finches, Lonchura punctulata. Behavioral Ecology and Sociobiology 34:251-256.

Giraldeau, L.-A., T. J. Valone, and J. J. Templeton. 2002. Potential disadvantages of using socially acquired information. Philosophical Transactions of the Royal Society B: Biological Sciences 357:15591566.

Ha, R. R., P. Bentzen, J. Marsh, and J. C. Ha. 2003. Kinship and association in social foraging northwestern crows (Corvus caurinus). Bird Behavior 15:65-75.

Held, S. D. E., R. W. Byrne, S. Jones, E. Murphy, M. Friel, and M. T. Mendl. 2010. Domestic pigs, Sus scrofa, adjust their foraging behaviour to whom they are foraging with. Animal Behaviour 79: 857-862.

Henzi, S. P., D. Lusseau, T. Weingrill, C. P. Schaik, and L. Barrett. 2009. Cyclicity in the structure of female baboon social networks. Behavioral Ecology and Sociobiology 63:1015-1021.

Hewitson, L., I. J. Gordon, and B. Dumont. 2007. Social context affects patch-leaving decisions of sheep in a variable environment. Animal Behaviour 74:239-246.

Houston, A. I., J. M. McNamara, and M. D. Steer. 2007. Do we 
expect natural selection to produce rational behaviour? Philosophical Transactions of the Royal Society B: Biological Sciences 362:1531-1543.

Huchard, E., A. Alvergne, D. Féjan, L. A. Knapp, G. Cowlishaw, and M. Raymond. 2010. More than friends? behavioural and genetic aspects of heterosexual associations in wild chacma baboons. Behavioral Ecology and Sociobiology 64:769-781.

Illius, A., P. Duncan, C. Richard, and P. Mesochina. 2002. Mechanisms of functional response and resource exploitation in browsing roe deer. Journal of Animal Ecology 71:723-734.

Johnson, C. A., L.-A. Giraldeau, and J. W. A. Grant. 2006. Intensity of interference affects the distribution of house sparrows, Passer domesticus, at food patches. Animal Behaviour 71:965-970.

Johnson, C. A., J. W. A. Grant, and L.-A. Giraldeau. 2004. The effect of patch size and competitor number on aggression among foraging house sparrows. Behavioral Ecology 15:412-418.

Kazahari, N., and N. Agetsuma. 2008. Social factors enhancing foraging success of a wild group of Japanese macaques (Macaca fuscata) in a patchy food environment. Behaviour 145:843-860.

King, A. J., F. E. Clark, and G. Cowlishaw. 2011. The dining etiquette of desert baboons: the roles of social bonds, kinship, and dominance in co-feeding networks. American Journal of Primatology 7:1-7.

King, A. J., and G. Cowlishaw. 2007. When to use social information: the advantage of large group size in individual decision making. Biology Letters 3:137-139.

2009. All together now: behavioural synchrony in baboons. Animal Behaviour 78:1381-1387.

King, A. J., N. J. B. Isaac, and G. Cowlishaw. 2009. Ecological, social, and reproductive factors shape producer-scrounger dynamics in baboons. Behavioral Ecology 20:1039-1049.

Krause, J., and G. D. Ruxton. 2002. Living in groups. Oxford University Press, Oxford.

Kurvers, R. H. J. M., H. H. T. Prins, S. E. van Wieren, K. van Oers, B. A. Nolet, and R. C. Ydenberg. 2010. The effect of personality on social foraging: shy barnacle geese scrounge more. Proceedings of the Royal Society B: Biological Sciences 277:601-608.

Lendvai, Á. Z., Z. Barta, A. Liker, and V. Bókony. 2004. The effect of energy reserves on social foraging: hungry sparrows scrounge more. Proceedings of the Royal Society B: Biological Sciences 271: 2467-2472.

Lukacs, P. M., K. P. Burnham, and D. R. Anderson. 2010. Model selection bias and Freedman's paradox. Annals of the Institute of Statistical Mathematics 62:117-125.

MacNulty, D. R., D. W. Smith, L. D. Mech, J. A. Vucetich, and C. Packer. 2012. Nonlinear effects of group size on the success of wolves hunting elk. Behavioral Ecology 23:75-82.

Manly, B., L. L. McDonald, D. Thomas, T. McDonald, and W. P. Erickson. 2002. Resource selection by animals: statistical design and analysis for field studies. 2nd ed. Kluwer, Dordrecht.

Mathot, K. J., and L.-A. Giraldeau. 2010. Within-group relatedness can lead to higher levels of exploitation: a model and empirical test. Behavioral Ecology 21:843-850.

McCracken, M. L., B. F. J. Manly, and M. V. Heyden. 1998. The use of discrete-choice models for evaluating resource selection. Journal of Agricultural, Biological, and Environmental Statistics 3:268-279.

McLoughlin, P. D., M. S. Boyce, T. Coulson, and T. H. CluttonBrock. 2006. Lifetime reproductive success and density-dependent, multi-variable resource selection. Proceedings of the Royal Society B: Biological Sciences 273:1449-1454.
Metz, M. C., J. A. Vucetich, D. W. Smith, D. R. Stahler, and R. O. Peterson. 2011. Effect of sociality and season on gray wolf (Canis lupus) foraging behavior: implications for estimating summer kill rate. PLoS ONE 6:e17332.

Milinski, M., and G. A. Parker. 1991. Competition for resources. Pages 137-168 in J. R. Krebs and N. B. Davies, eds. Behavioural ecology: an evolutionary approach. 3rd ed. Blackwell, Oxford.

Noldus Information Technology. 2003. Matman. Version 1.1.4. Noldus Information Technology, Wageningen.

Nooker, J. K., and B. K. Sandercock. 2008. Phenotypic correlates and survival consequences of male mating success in lek-mating greater prairie-chickens (Tympanuchus cupido). Behavioral Ecology and Sociobiology 62:1377-1388.

Quinn, J. L., and W. Cresswell. 2004. Predator hunting behaviour and prey vulnerability. Journal of Animal Ecology 73:143-154.

R Development Core Team. 2010. R: a language and environment for statistical computing. R Foundation for Statistical Computing, Vienna.

Rieucau, G., and L.-A. Giraldeau. 2011. Exploring the costs and benefits of social information use: an appraisal of current experimental evidence. Philosophical Transactions of the Royal Society B: Biological Sciences 366:949-957.

Rutten, A. L., K. Oosterbeek, S. Verhulst, N. J. Dingemanse, and B. J. Ens. 2010. Experimental evidence for interference competition in oystercatchers, Haematopus ostralegus. II. Free-living birds. Behavioral Ecology 21:1261-1270.

Sargeant, B. L., A. J. Wirsing, M. R. Heithaus, and J. Mann. 2006. Can environmental heterogeneity explain individual foraging variation in wild bottlenose dolphins (Tursiops sp.)? Behavioral Ecology and Sociobiology 61:679-688.

Silk, J. B. 2007. Social components of fitness in primate groups. Science 317:1347-1351.

Silk, J. B., J. C. Beehner, T. J. Bergman, C. Crockford, A. L. Engh, L. R. Moscovice, R. Wittig, R. M. Seyfarth, and D. L. Cheney. 2010a. Female chacma baboons form strong, equitable, and enduring social bonds. Behavioral Ecology and Sociobiology 64: 1733-1747.

- 2010b. Strong and consistent social bonds enhance the longevity of female baboons. Current Biology 20:1359-1361.

Sirot, E. 2000. An evolutionarily stable strategy for aggressiveness in feeding groups. Behavioral Ecology 11:351-356.

Smith, R., G. D. Ruxton, and W. Cresswell. 2001. Patch choice decisions of wild blackbirds: the role of preharvest public information. Animal Behaviour 61:1113-1124.

Stillman, R., J. Goss-Custard, R. Clarke, and S. E. A. le V. dit Durell. 1996. Shape of the interference function in a foraging vertebrate. Journal of Animal Ecology 65:813-824.

Symonds, M. R. E., and A. Moussalli. 2011. A brief guide to model selection, multimodel inference and model averaging in behavioural ecology using Akaike's information criterion. Behavioral Ecology and Sociobiology 65:13-21.

Templeton, J. J., and L.-A. Giraldeau. 1995. Patch assessment in foraging flocks of European starlings: evidence for the use of public information. Behavioral Ecology 6:65-72.

Tiddi, B., F. Aureli, E. Polizzi di Sorrentino, C. H. Janson, and G. Schino. 2011. Grooming for tolerance? two mechanisms of exchange in wild tufted capuchin monkeys. Behavioral Ecology 22: 663-669.

Tóth, Z., V. Bókony, Á. Z. Lendvai, K. Szabó, Z. Pénzes, and A. Liker. 
2009. Effects of relatedness on social-foraging tactic use in house sparrows. Animal Behaviour 77:337-342.

Train, K. 2003. Discrete choice methods with simulation. Cambridge University Press, Cambridge.

Valone, T. J., and J. J. Templeton. 2002. Public information for the assessment of quality: a widespread social phenomenon. Philosophical Transactions of the Royal Society B: Biological Sciences 357:1549-1557.

Waite, T. A., and K. L. Field. 2007. Foraging with others: games social
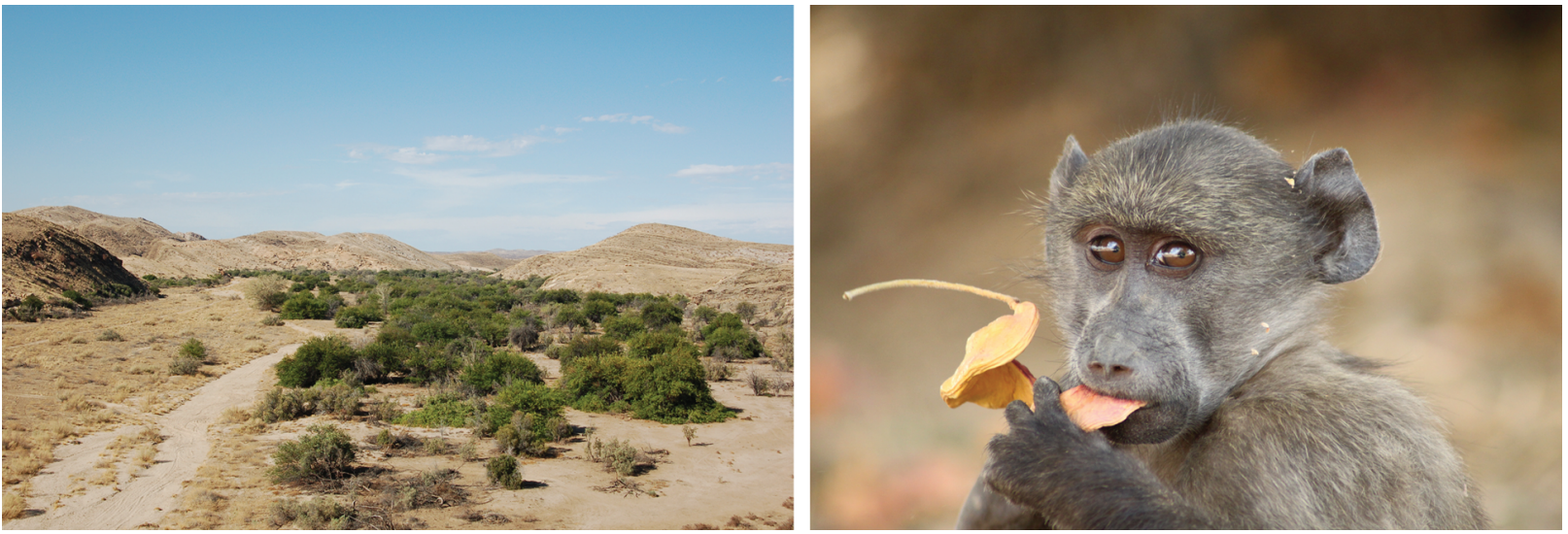

Left, the woodland and surrounding desert habitats at Tsaobis Leopard Park, Namibia (photo by Harry Marshall/Zoological Society of London [ZSL] Tsaobis Baboon Project). Right, infant chacma baboon Papio ursinus eats a Faidherbia albida pod (photo by Hannah Peck/ ZSL Tsaobis Baboon Project).

foragers play. Pages 331-362 in D. W. Stephens, J. S. Brown, and R. C. Ydenberg, eds. Foraging: behavior and ecology. University of Chicago Press, Chicago.

Wang, J. 2007. Triadic IBD coefficients and applications to estimating pairwise relatedness. Genetical Research 89:135-153.

Associate Editor: Jean-Michel Gaillard Editor: Judith L. Bronstein 
Copyright of American Naturalist is the property of University of Chicago Press and its content may not be copied or emailed to multiple sites or posted to a listserv without the copyright holder's express written permission. However, users may print, download, or email articles for individual use. 